DOI: $10.5216 /$ racs.v4.59219

\title{
Nuestro trabajo en las Milpas Educativas
}

\author{
María Bertely Busquets ${ }^{1}$
}

\section{RESUMEN}

Milpas Educativas es un proyecto colaborativo que tiene como objetivo construir un modelo educativo intercultural multilingüe, pertinente y relevante a las prácticas productivas, laborales y ceremoniales de las comunidades indígenas. El fundamento político, epistémico y pedagógico del proyecto es el Método Inductivo Intercultural (MII), que a lo largo de más de 20 años ha sido contextualizado a la realidad de las regiones indígenas mexicanas. Esta ponencia se centrará en las acciones que se están llevando a cabo en las Milpas Educativas para construir, registrar y discutir conocimientos territoriales y aprendizajes para el Buen Vivir de las comunidades involucradas en el proyecto. Nuestro objetivo es exponer de qué manera las acciones descritas permiten no sólo contribuir al proceso de formación pedagógica de las niñas y los niños indígenas, sino avanzar también hacia una educación crítica para la transformación social.

PALABRAS ClAVE: Educación intercultural. Milpas Educativas. Método Inductivo Intercultural. Buen vivir. Soberanía alimentaria.

\section{Nosso trabalho nas Milpas Educativas}

\section{RESUMO}

Milpas Educativas é um projeto colaborativo que visa construir um modelo educacional intercultural multilingue, pertinente e relevante para as práticas produtivas, de trabalho e cerimoniais de comunidades indígenas. A base política, epistêmica e pedagógica do projeto é o Método Indutivo Intercultural (MII), que ao longo de mais de 20 anos tem sido contextualizado para a realidade das regiões indígenas mexicanas. Este artigo enfocará as ações que estão sendo realizadas nas Milpas Educativas para criar, registrar e discutir o conhecimento territorial e a aprendizagem para o Bem Viver das comunidades envolvidas no projeto. Nosso objetivo é mostrar como as ações descritas permitem não somente contribuir para o processo de formação pedagógica das crianças indígenas, mas também avançar para uma educação crítica para a transformação social.

PALAVRAS-CHAVE: Educação intercultural. Milpas Educativas. Método Indutivo Intercultural. Bem viver. Soberania alimentar.

1 Profesora do Centro de Investigaciones y Estudios Superiores en Antropología Social (CIESAS). Ciudad de México, México. E-mail: articulandoeconstruindosaberes@gmail.com.

Dossiê Práticas de bem viver: diálogos possíveis entre o Núcleo Takinahakỹ e Milpas Educativas R. Articul.const.saber, 2019, v.4: e59219 


\section{Introducción}

En esta contribución expongo parte del trabajo que se realiza en el marco del proyecto Milpas Educativas, cuyo principal objetivo es construir, registrar y discutir conocimientos socionaturales encaminados a garantizar una vida buena para las comunidades involucradas. Antes de comenzar, conviene hacer una breve reseña de los antecedentes del proyecto y de los actores involucrados en el mismo. Es necesario remontarse al levantamiento zapatista en 1994, acontecimiento que despertó la conciencia de dignidad de los pueblos originarios, particularmente en las comunidades mayas de Chiapas, y la exigencia de justicia, transformación social y respeto a sus propios valores.

A partir de entonces, creció en los educadores comunitarios más jóvenes la inquietud de generar una propuesta educativa pertinente a sus contextos socionaturales así como a las actividades derivadas, a fin de revalorizar e incluir en las escuelas los conocimientos, el saberhacer y los significados indígenas. Es decir, una propuesta intercultural bilingüe y autonómica. Un grupo de esos educadores se organizó en 1995 y fundó la Unión de Maestros de la Nueva Educación de México (UNEM), que fue registrada legalmente como asociación civil en 1997. En 1996 la UNEM se acerca al Centro de Investigaciones y Estudios Superiores en Antropología Social (CIESAS) y varios de sus educadores recibieron capacitación en técnicas agroecológicas. Esos nuevos conocimientos se utilizaron gradualmente como estrategia pedagógica en las huertas escolares de las escuelas oficiales y autónomas en las que trabajaban.

Esos maestros comunitarios continuaron articulando en sus escuelas la teoría con la práctica pedagógica, mientras seguían formándose con María Bertely, del CIESAS, y Jorge Gasché, del Instituto de Investigaciones de la Amazonía Peruana (IIAP), dos expertos en el campo de la educación intercultural. El fundamento pedagógico de esa formación eran los principios del Método Inductivo Intercultural (MII) acuñado por Jorge Gasché, mismo que ha sido apropiado y contextualizado a la realidad de las regiones indígenas mexicanas por la UNEM a partir de la elaboración de diversos materiales educativos que se han producido a lo largo de más de 20 años.

Dossiê Práticas de bem viver: diálogos possíveis entre o Núcleo Takinahakỹ e Milpas Educativas

R. Articul.const.saber, 2019, v.4: e59219 
En 2007, la UNEM, con el acompañamiento de algunos académicos de diversas instituciones educativas, comenzó a impartir los diplomados Sistematización del conocimiento indígena y Diseño de materiales educativos interculturales y bilingües, dirigidos a profesores indígenas de nivel pre-escolar y primaria de los estados de Chiapas, Oaxaca, Puebla y Michoacán. Estos diplomados de formación docente recibieron continuidad como parte de un proyecto del CIESAS coordinado por María Bertely en el Laboratorio Lengua y Cultura Víctor Franco Pellotier. A partir de esa iniciativa se constituyó la Red de Educación Inductiva Intercultural (REDIIN), que incluye entre sus miembros a la UNEM y más de doscientos docentes indígenas y asesores mestizos, ampliándose hasta la región de Roraima, en Brasil.

Esta larga trayectoria política y de trabajo constituye las raíces en las que se ha venido edificando el proyecto Milpas Educativas que debemos entender como un proceso en construcción desde abajo sometido, por ello, a constantes tensiones y negociaciones.

En cuanto al Método Inductivo Intercultural (MII) podríamos definirlo, de forma muy somera, como una metodología educativa cuyas actividades pedagógicas se diseñan a partir de las mismas actividades sociales de las comunidades indígenas. Esta metodología permite explicitar y sistematizar los conocimientos comunitarios implícitos en esas actividades y articularlos con los conocimientos escolarizados.

Para facilitar la planeación de cada actividad didáctica el MII propone la llamada sintaxis cultural que representa la integración sociedad-naturaleza como un tejido de cuatro ejes temáticos presentes en cada actividad: territorio, recurso natural, trabajo o técnica y fin social. Esta sintaxis cultural queda caracterizada, a su vez, en la siguiente frase prototípica: "Vamos a nuestro territorio a pedir un recurso que transformaremos para satisfacer un fin social". Por tanto, para diseñar una actividad pedagógica específica es necesario concretizar esa frase, por ejemplo: "Vamos a la milpa (territorio) para cosechar (técnica) lo que ha producido (producto) y tener comida para nuestra familia (fin social)"”.

Ahora bien, ¿en qué consiste, en concreto, el proyecto Milpas Educativas? Se trata de un proyecto interinstitucional coordinado desde el Instituto de Investigaciones para el Desarrollo de la Educación (INIDE) de la Universidad Iberoamericana, en la persona de Stefano Sartorello, y desde el Centro de Investigaciones y Estudios Superiores en Antropología Social (CIESAS), en la persona de María Bertely.

Dossiê Práticas de bem viver: diálogos possíveis entre o Núcleo Takinahakỹ e Milpas Educativas R. Articul.const.saber, 2019, v.4: e59219 
Con este proyecto colaborativo, fundamentado en el MII, comuneros, niñas y niños indígenas, educadores comunitarios, maestros oficiales, académicos y científicos de diferentes instituciones generan:

- una práctica educativa alternativa que revaloriza y empodera a la sociedad indígena en diversas regiones del país, confiando en sus capacidades y liberando su creatividad

- aprendizajes interculturales útiles para la construcción de una vida buena en el territorio socionatural comunitario, de acuerdo con los proyectos sociales y planes de vida de las familias y comunidades participantes.

El proyecto es financiado por la Fundación W.K. Kellogg y se desarrolla en 31 comunidades indígenas de diversas regiones del país. Otra veintena de milpas se encuentra en proceso de instalación. Las Milpas Educativas son espacios vivos y situaciones reales en el territorio socionatural de una comunidad en el que las y los comuneros realizan actividades que generan aprendizajes para el Buen Vivir. Se puede hacer milpa en una escuela, un río, un solar o un monte para enseñar y aprender mediante actividades sociales, productivas, curativas, rituales, entre otras. En ellas, la tradición oral y las prácticas vivenciales indígenas se incorporan al currículo junto con las actividades pedagógicas en las aulas y los conocimientos empíricos de los comuneros en campos como la agroecología, la soberanía alimentaria, la alimentación sana y la salud comunitaria. Una educación intercultural de esta naturaleza integra la escuela a la vida comunitaria y propicia un mayor arraigo comunitario mediante el inter-aprendizaje y la realidad vivencial en el territorio comunitario.

\section{Un proyecto intercultural centrado en el Buen Vivir y la soberanía alimentaria}

Desde su inicio, los coordinadores de las Milpas Educativas sabían que el proceso de creación de la propuesta educativa para las milpas, además de explicitar y sistematizar los conocimientos propios de las sociedades indígenas y articularlos con los conocimientos escolarizados, debía avanzar hacia una educación crítica para la transformación social. En eso consiste principalmente el trabajo en las Milpas Educativas, en proponer y acompañar acciones

Dossiê Práticas de bem viver: diálogos possíveis entre o Núcleo Takinahakỹ e Milpas Educativas

R. Articul.const.saber, 2019, v.4: e59219 
para: sentipensar de forma colectiva el territorio, sus riquezas, pero también sus afectaciones y amenazas. A la vez, al estar en las Milpas se contrastan los valores que refleja la sociedad dominante con los valores de una sociedad alternativa, propia de los pueblos originarios, preocupada por una vida buena y justa. En adelante se describen cuáles son y en qué consisten las acciones que hemos propuesto para alcanzar ambos objetivos.

Como ya se ha mencionado, el propósito central del proyecto Milpas Educativas es generar una metodología para mejorar la pertinencia y relevancia de los procesos educativos formales e informales de niñas y niños indígenas. Con esto en mente, todos los participantes involucrados, educadores comunitarios, acompañantes mestizos y comuneros, se comprometieron a generar actividades educativas para cada una de las comunidades. Además de articular los conocimientos propios con los escolarizados, estas actividades debían abonar al Buen Vivir y la soberanía alimentaria, para lo cual se levantan registros fotográficos y notas que permiten extraer estas enseñanzas.

Aquí conviene detenerse un momento para precisar, a partir de las observaciones de los compañeros de las comunidades de Chiapas, qué se define en las Milpas Educativas por Buen Vivir.

En un principio la referencia principal fueron los textos sobre la noción tseltal de lekil kuxlejal estudiada por el sociólogo y comunicólogo José Antonio Paoli Bolio, quien la define como "la vida buena por antonomasia" (Paoli, 2001). Desde esta perspectiva el Buen Vivir se define como un ideal de vida, un mundo bueno como aspiración y que, en gran medida, ha dejado de ser. Sin embargo, cuando compartimos esta noción con los educadores y los expertos tsotsiles, tseltales y ch'oles de las comunidades de Chiapas, ninguno recordaba que, en lengua indígena, sus padres o abuelos agreraran al sentido de la vida los adjetivos de "buena" o "mala".

Muchos de ellos pensaban que se trataba de un término construido desde una perspectiva occidentalizada que, además, suponía algunas incongruencias con el proyecto que es incluyente, pues, como nos señaló el colaborador tsotsil Elías, doctor en lingüística, tenía asociada una concepción moralista porque califica lo que es el bien y el mal. Lekil kuxlejal da a entender también que es el resultado de una acción, no indica un proceso, mientras que una milpa es un proceso, no es algo acabado, ni bueno ni malo, es algo vivo que se va haciendo poco a poco.

Dossiê Práticas de bem viver: diálogos possíveis entre o Núcleo Takinahakỹ e Milpas Educativas R. Articul.const.saber, 2019, v.4: e59219 
Pero ¿de qué manera vamos a explicitar los conceptos, conocimientos y valores socionaturales propios relacionados con el Buen Vivir e implícitos en la actividad? Para ello consideraremos los cuatro ejes de la sintaxis cultural para formular reflexiones e interaprendizajes relacionados con el Buen Vivir, la soberanía alimentaria, las riquezas, devastaciones y resistencias territoriales.

El MII hace uso de una sintaxis cultural, antes mencionada, como punto de partida de sus contenidos y actividades pedagógicas. En este caso, nuestro diagnóstico socionatural partirá de la misma sintaxis para satisfacer los siguientes objetivos:

1. Producir conceptos, conocimientos y valores territoriales alternativos que cuestionen las versiones dominantes de pobreza, desarrollo y modernidad.

2. Educar para el ejercicio de la autonomía y la auto-determinación de los pueblos indígenas, así como su derecho a participar en la construcción de una educación que asegure el Buen Vivir en territorios propios.

3. Actuar en contra de la fragmentación y privatización territorial así como los daños causados a la Madre Tierra a consecuencia del mal gobierno, las políticas extractivas y los intereses ajenos a las comunidades.

4. Elaborar, de manera comunal y participativa, diagnósticos socionaturales a partir de las actividades que den cuenta de la integralidad que engloba al territorio indígena.

De modo que una premisa del trabajo en las Milpas Educativas consiste en el reconocimiento de los conocimientos y significados locales que han jugado un rol trascendental en la permanencia y continuidad de la vida campesina y comunitaria así como en la necesidad de conservarlos y promoverlos para resistir el embate de la sociedad occidental con su frenético productivismo y consumismo. No obstante, sostenemos también que la justicia epistemológica está íntimamente unida a la justicia social, por lo que además de la dimensión pedagógica, la propuesta de educación intercultural que se está sembrando en las Milpas Educativas considera las dimensiones ontológica, política, pedagogica y axiológica. Sobre esas cuatro dimensiones se asienta, a fin de cuentas, el perfil de egreso definido por los maestros de la 
UNEM y sus colaboradores mestizos en su modelo curricular de educación intercultural bilingüe y son, además, los principios que rigen el MII.

\section{Primera línea de trabajo: la fotovoz}

Nuestra primera línea de trabajo en las Milpas Educativas consiste en conciliar los requerimientos pedagógicos de la propuesta educativa con los planteamientos políticosfilosóficos que abonan a "la construcción de nuevas formas de ciudadanía étnica e intercultural" (Sartorello, 2016, p. 156). En otras palabras, lo que buscamos al proponer ampliar las reflexiones e interaprendizajes para que a la sintaxis cultural no opere sólo como una "red para pescar contenidos [escolares] de las actividades sociales" (Gasché, 2006, citado en AAVV, 2009, p. 95), sino que permita, además, pescar conocimientos territoriales y culturales que caracterizan a un tipo de sociedad diferente a la occidental. Una sociedad que tiene diferentes nociones del tiempo, del espacio, y que establece otro tipo de relaciones con los seres espirituales y de la naturaleza.

En este proyecto, como podremos comprobar en los artículos de la Red de Educación Inductiva Intercultural, México, incluidos en este número temático, la generación y el registro de los conocimientos territoriales relacionados con el Buen Vivir, la soberanía alimentaria, las riquezas, las afectaciones y las resistencias territoriales son resultado de un proceso colaborativo, dialógico y horizontal. Contrariamente a lo que constituye la investigación científica en la que una persona o grupo investigador que se califica como calificado o experto extrae el conocimiento de lo que le rodea y detecta posibles situaciones a mejorar o cambiar, en este caso, las y los educadores y las y los comuneros, niñas y niños debían participar de manera activa en el proceso de investigación y creación de conocimientos territoriales que puede aportar una actividad pedagógica específica. Por esa razón la forma de indagación que apoyó parte del trabajo en las Milpas Educativas fue similar a la Investigación-Acción-Participativa, que cuestiona la supuesta neutralidad y objetividad de los investigadores y propone que sean las personas afectadas por un problema quienes participen activamente en la transformación y creación de conocimiento e intervención sobre su realidad. Pero solo fue similar porque el MII implica necesariamente la interculturalidad, el encuentro entre indígenas y no indígenas, y entre indígenas, y se enfoca, en específico, a propiciar interaprendizajes y develar los conocimientos

Dossiê Práticas de bem viver: diálogos possíveis entre o Núcleo Takinahakỹ e Milpas Educativas

R. Articul.const.saber, 2019, v.4: e59219 
implícitos en actividades comunitarias, técnicas locales y recursos vegetales y animales no considerados fuentes de conocimiento por estar sometidos al desprecio así como a mecanismos de dominación objetivos y subjetivos.

No obstante el protagonismo que adquieren las comunidades y usuarios tanto en la Investigación-Acción-Participativa como en el MII, este último da lugar a una definición alternativa de la educación intercultural, ya no como anhelo o aspiración o como un diálogo entre culturas factibles de complementarse, sino como una experiencia de interaprendizajes, que se da través de actividades, que permite explicitar valores, conocimientos y significados locales indígenas y/o campesinos-, que provocan en los agentes externos -académicos, urbanitas, "mestizos", no indígenas- sorpresas, rupturas epistémicas, crisis ontológicas, dudas sobre la "validez" de sus propias certezas. Trabajar con el MII implica conflictos entre los enfoques científicos occidentales, que buscan principios y leyes explicativas, y los corpus de conocimientos situados y contextualizados, derivados de la realización de una actividad práctica que depende de la integridad Sociedad-Naturaleza. En consecuencia, la educación intercultural, desde un enfoque decolonial, implica reconocer por qué y cómo las condiciones que intervienen en la realización de la actividad son siempre cambiantes, en tiempos y espacios configurados a partir de la integridad Sociedad-Naturaleza.

“¿Cómo preparar atole de maíz tierno?”. "todo depende”, dirían las y los comuneros. Preguntarían: “de quién y cómo es el terreno donde nació el maíz?”, “¿a qué altura se prendió la fogata?”, “se cocinará en olla de barro o metal?”, “¿qué leña se usará?”, “¿cómo se encuentra la luna, tierna o madura?”, ¿cómo está el clima?”, “¿está o no embarazada quien prepara el atole?”, “¿el rezo o bendiciones pueden “curar” los “males” adquiridos por el atole?”. Además, nada es basura o desperdicio, porque aún la masa que se desborda del moledor de maíz, y cae al piso, es para los pollos.

Aclarado el enfoque metodológico, las herramientas y técnicas utilizados se apoyan en medios audiovisuales con la finalidad de que todos los miembros de la comunidad que así lo deseen, puedan participar. Los métodos audiovisuales resultan menos agresivos e invasivos que una etnografía basada en encuestas y entrevistas para la obtención de información y permiten a

Dossiê Práticas de bem viver: diálogos possíveis entre o Núcleo Takinahakỹ e Milpas Educativas

R. Articul.const.saber, 2019, v.4: e59219 
las personas que no están o han abandonado la escuela participar en el proceso aportando sus propios conocimientos. Nosotros optamos por "fijar" tanto los procesos como los aprendizajes tenidos en las actividades por medio de una técnica conocida como fotovoz. Esta consiste en combinar la fotografía - texto icónico - con una pequeña historia - texto escrito - del proceso y los aprendizajes que la misma fotografía sugiere. Es, por tanto, una técnica adecuada para construir en colectivo el diagnóstico socionatural planteado como objetivo que requiere la colaboración activa de la comunidad.

En lugar de que los colaboradores externos elaboren un cuestionario de preguntas e indicadores predefinidos, la sintaxis cultural del MII articula los conocimientos, valores y recursos de cada comunidad. Son las y los comuneros mismos, los que en verdad conocen su territorio, en compañía de niñas y niños, en interaprendizaje, los que documentarán sus propias realidades y elaborarán el diagnóstico. Esta técnica, como herramienta de resistencia, permite utilizar las nuevas Tecnologías de la Información y Comunicación (TICs) para generar nuevos conocimientos respetuosos de la cosmovisión y realidad indígena. Las fotografías se están tomando en cada Milpa, de tal manera que, mientras algunos de los participantes están realizando la actividad, otros están haciendo el registro para la fotovoz.

Realizar el auto-diagnóstico de los conocimientos para la vida buena que se deben enseñar y aprender en las Milpas implica trabajar sobre las cuatro dimensiones de la sintaxis cultural que, de hecho, son las cuatro dimensiones del currículum escolar: ontológica, política, axiológica y pedadógica. En diversos Talleres Regionales Intensivos se fue perfilando de manera colectiva la forma en la que la fotovoz sería utilizada en las Milpas Educativas, para registrar y representar las experiencias y los inter-aprendizajes para el kuxlejal, así como las acciones de resistencia frente al poder egoísta que se están dando, de manera cotidiana, en el territorio socionatural comunitario. Así pues, en las fotovoces, los investigadores nos convertimos en meros facilitadores del proceso.

En los primeros talleres fueron precisándose los aspectos de cada una de las cuatro dimensiones que podrían abordarse mediante la fotovoz. Para la dimensión ontológica la reflexión se encaminó hacia la Integración Sociedad-Naturaleza, que es sin duda la característica distintiva de la sociedad indígena que procura una relación armoniosa con la madre tierra. No habrá una buena vida, no se alcanzará el kuxlejal si no hay armonía con la Madre Tierra que los 
cuida y los alimenta. Esta armonía sociedad-naturaleza se manifiesta constantemente en las actividades cotidianas de la comunidad.

En cuanto a la dimensión política, la cuestión sobre la que se consideró necesario reflexionar con fotovoz fue la resistencia frente al avasallamiento del neoliberalismo y su modelo extractivista, con acciones como la autosuficiencia, la soberanía alimentaria, la economía solidaria, la revalorización de los conocimientos comunitarios y las luchas étnico-territoriales en defensa del territorio. Dentro de la dimensión axiológica, lo que destaca es la necesidad de conocer los fines y valores de una actividad específica como son subsistencia, protección, afecto, respeto, participación, solidaridad, recreo, identidad y autonomía.

Finalmente, como ya se mencionó, en la dimensión pedagógica las fotovoces deben resaltar la relación entre el Método Inductivo Intercultural, la realización de la actividad concreta y el kuxlejal. La fotovoz y la reflexión que ésta detona se concentra en el aporte de la actividad social, productiva, recreativa o ritual a la vida buena y las consecuencias que tiene continuar o dejar de practicarla. Además debe mostrar qué impacto tendría dañar alguno de los ejes de la sintaxis cultural: si dañamos el territorio, si acabamos con alguno de los recursos naturales necesarios para la actividad, si nos olvidamos del trabajo y la técnica transmitida por los abuelos y lo sustituimos por el trabajo fácil y la tecnología occidental, o si dejamos de lado las actividades que persiguen la satisfacción de nuestras necesidades de manera autosuficiente y autogestiva.

Pero como nos enseñaron algunas maestras de Puebla, la fotovoz también puede convertirse en un recurso para mostrar un "ciclo de actividades" que se articulan mediante el Calendario Socionatural, que persiguen un fin mediato y específico. De este modo, las maestras están produciendo insumos para el comedor escolar mientras, al mismo tiempo, sintetizan las distintas actividades realizadas en milpas familiares, escolares y comunitarias. Aunque las Milpas Educativas cuentan con otros espacios de reflexión conjunta sobre los modos de aprender pertinentes y de compartir conocimientos que promueve el MII, con la fotovoz los educadores comunitarios, los comuneros y los niños y niñas pueden generar fotografías y narrativas que cumplan con los objetivos de este proyecto.

Sin lugar a dudas, lo más importante de una metodología como fotovoz no es el producto en sí mismo, sino el proceso de reflexión y de diálogo colectivo que ha de llevarnos a alcanzar 
nuestro objetivo común: mostrar nuestro trabajo para la salud, la autosuficiencia alimentaria y la vida en las Milpas Educativas. Con esto en mente, derivado de las primeras actividades para el kuxlejal documentadas con fotovoz, establecimos la siguiente tipología de fotovoces:

- $\quad$ Secuencias de una actividad con los recursos que nos regala la milpa

- $\quad$ Secuencias de actividades que se van tejiendo en un ciclo anual, mediante el Calendario Socionatural y en torno a fines sociales específicos (la pesca) o generales (comedor escolar)

- $\quad$ De niñas y niños para niñas y niños

- $\quad$ Procesos históricos y cambios en una actividad: valores, conocimientos y haceres de abuelas y abuelos

- $\quad$ Procesos históricos y afectaciones territoriales

- Milpas demostrativas de sanación territorial

- $\quad$ Actividades de reciclaje e impactos positivos y negativos en nuestras milpas

- Cómo se puede fortalecer el Bien Común en contextos escolarizados, urbanos, industrializados y en conflicto

\section{Segunda línea de trabajo: mapas vivos}

La segunda línea de trabajo emergente que se ha construido colectivamente a lo largo de esta primera etapa de las Milpas Educativas tiene como finalidad ayudar a problematizar el territorio en el que se ha instalado alguna milpa. En los diversos Talleres Regionales Intensivos se discutió ampliamente la necesidad que tienen las comunidades de conocer bien su realidad espacial y social para poder defender sus derechos, descubrir conflictos y problemas de la comunidad y generar acciones de resistencia territorial. En concreto, esta línea de trabajo consiste en generar "mapas vivos" como una herramienta de defensa del territorio que se habita y como forma de resistencia ante los problemas que se están dando debido al acaparamiento de tierras con grandes recursos naturales bajo el modelo extractivista.

Dossiê Práticas de bem viver: diálogos possíveis entre o Núcleo Takinahakỹ e Milpas Educativas

R. Articul.const.saber, 2019, v.4: e59219 
A diferencia de los mapas convencionales, que son elaborados por técnicos considerados expertos en la representación espacial, los mapas vivos son registros visuales de un territorio construidos de manera colectiva y horizontal. En este caso, el territorio no se limita a la superficie terrestre, sino que incluye el modo de vida y la sabiduría que las comunidades comparten. Desde esta perspectiva, consideramos que los mapas vivos pueden abarcar información muy diversa, según las necesidades de cada momento y lugar así como:

- Representar la cultura y los valores positivos de la sociedad indígena

- Visibilizar el contraste entre dos tipos de sociedad indígena/occidental

- Mostrar las transformaciones del territorio a lo largo del tiempo:

- Mapa del pasado para reconocer el territorio ancestral.

- Mapa del presente con todos sus problemas socionaturales.

- Mapa del futuro que muestre cómo quiere vivir y a dónde quiere llegar la comunidad.

- Mapeo de autoría nativa que refleje cómo estamos sembrando nuestras semillas del kuxlejal.

Como materiales complementarios, se recurre a los Sistemas de Información Geográfica (SIG) para elaborar mapas que permitan visualizar los avances del trabajo en milpas activas y milpas por sembrarse con el propósito de observar los frutos de nuestro trabajo, en atención a experiencias emergentes. Así mismo el SIG nos permitió mapear la devastación territorial producida por los megaproyectos extractivistas y las resistencias emprendidas en defensa del territorio.

Una vez se han hecho los mapas con las temáticas acordadas con la comunidad, estos documentos pueden tener diferentes aplicaciones. Una de las más mencionadas en los talleres realizados fue su utilidad como instrumento de alfabetización ética, territorial y jurídica que germina y crece en la vida práctica.

Milpas Educativas es un proyecto que cuenta con una visión amplia de la educación y pedagogía, se trata también de promover la defensa de la vida, una ética del Buen Vivir, la integración armónica sociedad-naturaleza y el trabajo colectivo frente al individualismo capitalista. Es a partir de esta convergencia y estas reflexiones, presentes en el proyecto Milpas

Dossiê Práticas de bem viver: diálogos possíveis entre o Núcleo Takinahakỹ e Milpas Educativas R. Articul.const.saber, 2019, v.4: e59219 
Educativas, que surgió la motivación por aprovechar los inter-aprendizaje para generar, desde el inicio de nuestros trabajos y en diálogo con la comunidad, Planes de Vida.

Los planes de vida se inspiran en una de las iniciativas desplegadas por el Consejo Regional Indígena del Cauca (CRIC), organización indígena del departamento del Cauca en Colombia, como un proyecto de vida de los pueblos indígenas para la defensa de sus territorios, sus lenguas y sus culturas. Los planes de vida serán presentados por autoridades, expertos y expertas locales, padres y madres de familia de las comunidades, y profesores y profesoras durante el 2019. Se espera que incluyan los aprendizajes para el Buen Vivir, la soberanía alimentaria, así como los relacionados con el descubrimiento de las riquezas, riesgos y afectaciones territoriales, derivados de las actividades educativas realizadas en el territorio y, como parte de estos aprendizajes, los conocimientos socionaturales relacionados. Tales aprendizajes, sumados a los diálogos entre los dos tipos de sociedad, darán cuenta, entre otras cosas, de la visión propia de cada comunidad acerca de:

- una relación armónica de la sociedad con la naturaleza

- $\quad$ una educación y un servicio de salud pertinentes

- una vivienda digna

- una dieta nutritiva y balanceada

\section{A modo de conclusión}

Por todo lo explicado hasta ahora, es posible concluir que esta propuesta atiende dos finalidades:

1. Hacer visible, desde una mirada propia, la pluralidad de caracterizaciones y vivencias que cada comunidad tiene de su territorio en contraste con la visión deformada que tiene de ésta la sociedad dominante.

2. Conocer los aprendizajes para el Buen Vivir que las y los niños, comuneros, educadores y acompañantes académicos lograron a través del Método Inductivo Intercultural.

Dossiê Práticas de bem viver: diálogos possíveis entre o Núcleo Takinahakỹ e Milpas Educativas

R. Articul.const.saber, 2019, v.4: e59219 
Hasta ahora, el proyecto ha generado:

Producto 1: Documentación de actividades que muestran los procesos de planeación, operación y evaluación de los aprendizajes familiares y comunitarios que se dan en las Milpas Educativas, incluidos los de las niñas y los niños de educación inicial, preescolar y primaria.

Producto 2: Fotovoz de una actividad, incluidos los aprendizajes derivados del trabajo pedagógico en los cuatro ejes de la sintaxis cultural del MII. Con este producto se mostrarán los aprendizajes relacionados con el Buen Vivir, la soberanía alimentaria, las riquezas, los riesgos y las afectaciones territoriales.

Producto 3: Mapas vivos de la resistencia pasada, presente y futura que reflejen cómo estamos sembrando nuestras semillas del kuxlejal.

Producto 4: Testimonio audiovisual de Planes de Vida por comunidad o pueblo indígena. Estos se devolverán, con los demás productos, a las comunidades participantes.

Los objetivos, trabajos y productos propuestos buscan brindar elementos para construir, de forma colaborativa y respetuosa, principios políticos, epistémicos y pedagógicos que reflejen la filosofía de vida del Buen Vivir. Si se pretende fomentar el arraigo sociocultural en lugar de la migración en las Milpas Educativas, resulta obligado establecer procesos en que los comuneros y educadores analicen de manera crítica los conceptos occidentales de desarrollo, calidad de vida, eficacia, economía, entre otros y su incidencia positiva y/o negativa en las culturas indígenas.

Así mismo, no puede incentivarse el arraigo sociocultural de los más jóvenes, sin antes revisar los procesos socioculturales propios y la integración Sociedad-Naturaleza de cada comunidad que fundamentan su identidad cultural. En función de esta caracterización y alfabetización territorial, las comunidades indígenas buscan construir sociedades igualitarias, destinadas a promover el Buen Vivir para todos.

Dossiê Práticas de bem viver: diálogos possíveis entre o Núcleo Takinahakỹ e Milpas Educativas R. Articul.const.saber, 2019, v.4: e59219 


\section{Referencias Bibliográficas}

AAVV. Modelo curricular de educación intercultural bilingüe UNEM. En M. Bertely (Coord.), Sembrando nuestra educación intercultural como derecho: Diálogos, experiencias y modelos educativos de dos organizaciones indígenas del estado de Chiapas. Ciudad de México: UNEM/ECIDEA/CIESAS/Papeles de la Casa Chata/IIAPOEI/Ediciones Alcatraz. 2009.

Bertely, M., Gasché, J. y Podestá, R. (Coords.). Educando en la diversidad: Investigaciones y experiencias educativas interculturales y bilinguies. Quito: Abya-Yala. 2008.

Bonfil, B. G. México profundo: Una civilización negada. Ciudad de México: Secretaría de Educación Pública. 1987.

Gasché, J. Taller de diseño curricular en Ocosingo. Chiapas, México. 2006.

Paoli, A. Lekil kuxlejal: Aproximaciones al ideal de vida entre los tseltales. Revista Chiapas, 12, 149-164. 2001.

Sartorello, S. La co-teorización intercultural de un modelo educativo en Chiapas, México. Quito: Abya-Yala. 2016.

Submetido em 27 de junho de 2019.

Aceito em 08 de agosto de 2019.

Publicado em 09 de agosto de 2019.

Dossiê Práticas de bem viver: diálogos possíveis entre o Núcleo Takinahakỹ e Milpas Educativas

R. Articul.const.saber, 2019, v.4: e59219 